\title{
Impact of retransfusion of blood processed in cell-saver on coagulation versus cardiopulmonary bypass: A prospective observational study using thromboelastography
}

\author{
Tomas Vymazal, Martin Filaun, Michal Horacek
}

\begin{abstract}
Aims. To compare an impact of retransfusion of blood processed in cell-saver (CS) with that of cardiopulmonary bypass (CPB) on blood coagulation in patients undergoing cardiac surgery.

Methods. Prospective observational study using thromboelastography (TEG).

Results. TEG samples from 170 patients were analyzed. Cardiopulmonary bypass was used in 100 patients while 70 patients were operated off-pump. In 20 off-pump patients collected blood was processed by cell-saver and returned. In all patients clot formation after heparin neutralization by protamine was unimpaired. However, there was a significant increase in fibrinolysis defined by the TEG parameter Lysis time $30 \mathrm{~min}$ after the maximum amplitude of the clot was reached (Ly30) in groups with CPB or CS but this increase still did not exceed the threshold for clinical fibrinolysis (Ly30 $>7.5 \%$ ). In the group without CPB there was no significant impact on coagulation.
\end{abstract}

Conclusion. Surgery that avoids CPB and/or CS is the gentlest method for inducing blood coagulation.

Key words: thromboelastography, coagulopathy, bleeding, cardiopulmonary bypass, cell-saver, fibrinolysis

Received: November 5, 2012; Accepted: February 14, 2013; Available online: March 22, 2013

http://dx.doi.org/10.5507/bp.2013.012

Department of Anaesthesiology and Intensive Care Medicine, $2^{\text {nd }}$ Medical School, Charles University in Prague and University Hospital Motol, Prague, Czech Republic

Corresponding author:Tomas Vymazal, email: tomas.vymazal@fnmotol.cz

\section{INTRODUCTION}

Cardiac surgical procedures are complex, usually longlasting and performed most frequently in seniors suffering from concomitant diseases and taking various medications. The majority of procedures are accomplished on cardiopulmonary bypass (CPB) but some can also be performed without $\mathrm{CPB}$ on the beating heart. These are offpump coronary artery bypasses (OPCAB). In all coronary revascularizations, the decision on how to perform the procedure in a particular patient belongs to the operating surgeon. Principally it is possible to do the case either on pump or on beating heart.

Cardiac surgical procedures carry the risk of considerable bleeding which is caused by surgical wound itself and by heparin and protamine together with other causes such as hypothermia, anaemia, inflammation or drug effects (e.g. antiplatelet agents, low molecular weight heparins) etc. To reduce the burden associated with blood transfusions - transfusion-associated immunomodulation, lung injury or circulatory overload to name just a few - resulting in decreasing patients' survival with increasing number of transfusions ${ }^{1}$ the shed blood in pump procedures is often retransfused directly back to the patient or in offpump procedures it is collected to a cell-saver, processed and also returned back to the patient. In both cases, the blood is in contact with a serose lining in the pericardium and/or in pleural cavities, sometimes for a long time. It has been shown that blood contact with the pericardial cavity induces strong hemolysis, inflammatory mediator release, and coagulation activation, driven by tissue factor pathway activation ${ }^{2}$.

Therefore, the aim of this prospective observational study was to compare the impact of cardiopulmonary bypass plus retransfusion of the shed blood with retransfusion of shed blood processed by a cell-saver in off-pump procedures. The impact on coagulation was assessed thromboelastographically. The hypothesis was that retransfusion of blood from cell-saver could impair coagulation equally as cardiopulmonary bypass.

\section{MATERIAL AND METHODS}

\section{Patient sample}

The study was done in adult patients undergoing cardiac surgical procedures including coronary artery bypass grafting on pump (CABG) and off-pump (OPCABG) and/or valve procedures at a university hospital between June 2010 and June 2011. To evaluate the impact of both approaches (i.e. direct retransfusion on pump vs. retransfusion from cell-saver in off-pump procedures) on coagulation, thromboelastography (TEG) was used. All the relevant data for analysis were obtained from cardiac anaesthesia and surgical intensive care databases collected prospectively during the patient's hospitalization as a part of routine clinical practice. Therefore, individual consent was waived as this was a retrospective analysis of prospectively gathered data and patients were not identified. 


\section{Anaesthesiological and coagulation management}

All anaesthetic and surgical procedures were performed in the standard manner. General anesthesia was principally the same in on-pump and off-pump procedures. It was induced by a combination of sufentanil 1 $\mu \mathrm{g} . \mathrm{kg}^{-1}$, etomidate $0.2 \mathrm{mg} \cdot \mathrm{kg}^{-1}$ and rocuronium $1 \mathrm{mg} . \mathrm{kg}^{-1}$ and maintained by isoflurane in air and boluses of sufentanil and rocuronium being supplemented as needed.

Before connecting the patient to the CPB 3-4 mg. $\mathrm{kg}^{-1}$ body weight bovine lung heparin were given into the central vein to achieve an activated coagulation time (ACT) at least $400 \mathrm{~s}$. The standard cardiopulmonary bypass with open venous reservoir, twin-head roller pumps, capillary membrane oxygenator, and arterial line filtration and biocompatible substances coated circuit was performed using mild hypothermia $34-35^{\circ} \mathrm{C}$. A cold blood antegrade and/ or retrograde cardioplegia were applied for myocardial protection according to surgeon's discretion. After successful weaning from CPB protamine was given in a dose of $1 \mathrm{mg}$ per $\mathrm{mg}$ of administered heparin into a central vein to achieve ACT less than $140 \mathrm{~s}$. Inotropic and vasoactive drugs were supplemented as needed. Aprotinin or antifibrinolytics were not routinely used during the study.

In off-pump procedures, heparin was given in a dose 2-3 mg. $\mathrm{kg}^{-1}$ body weight into the central vein on surgeon's request. The desired ACT value was at least $300 \mathrm{~s}$. The anastomosis of left internal mammary artery to left anterior descending artery was constructed first, other anastomoses were then performed using stabilizers at the surgeon's discretion. Necessary hemodynamic support was achieved with volume, inotropic agents and cardiac pacing as needed. After completing the last anastomosis when circulation was stable, protamine was given in the same ratio to heparin as in on-pump procedures.

Cell-saver (a continuous autotransfusion system C.A.T.S. Fresenius Medical) was routinely used in all off-pump procedures since their beginning respecting its contraindications such as sepsis and malignant tumors. In all off-pump cases, the lost blood was suctioned into the reservoir of the cell-saver where heparin in a dose $50 \mathrm{mg}$ was added. However, the collected blood was processed only if the blood loss was more than 1 liter and/or if the patient was anaemic and the amount of blood gained after processing (roughly one third of blood collected) was high enough to justify the costs of additional instrumental setting. The processed blood was retransfused to patients immediately after processing in accordance with circulatory condition.

\section{Thromboelastography}

Thromboelastography was performed in the standard manner according to the manufacturer's manual using a computer-controlled thromboelastograph haemostasis system (TEG Haemoscope, Niles, Illinois, USA) in native blood samples withdrawn from arterial lines after discarding $10 \mathrm{ml}$ of blood to eliminate the effect of heparin flush because all arterial lines were continuously flushed with a heparin solution ( 4 IU.mL $\mathrm{mL}^{-1}, 3 \mathrm{~mL} \cdot \mathrm{h}^{-1}$ ). The TEG analysis was done at the temperature $37^{\circ} \mathrm{C}$. There were two samples for each patient. The first sample was taken after the induction of anaesthesia before heparin administration, the second one in patients operated on pump within 20 min after coming off the bypass and after heparin neutralization by protamine or in off-pump patients after retransfusion the blood gained from the cell-saver and again after heparin neutralization by protamine. The samples were compared within and between the groups.

Recorded values from thromboelastography were $\mathrm{R}$ (reaction time), i.e. time from the start of the sample run to the first detectable fibrin fiber formation defined by curve amplitude width $2 \mathrm{~mm}$ (normal range 19-28 mm); $\mathrm{K}$ (clotting time) is a time from $\mathrm{R}$ to the arbitrarily predefined strength of the clot, i.e. the width of the TEG trace $20 \mathrm{~mm}$ (normal range $8-13 \mathrm{~mm}$ ); MA, maximum clot amplitude (normal range $48-60 \mathrm{~mm}$ ); LY30 is an amplitude decrement measured 30 min after the MA expressed in \% of MA (normal range $<7.5 \%$ ). The tracing speed was $2 \mathrm{~mm} \cdot \mathrm{min}^{-1}$. Reaction time R corresponds to the whole blood clotting time. Values $\mathrm{R}, \mathrm{K}$ and $\alpha$-angle (not collected) indicate the rate of clot formation whereas MA represents the strength of the blood clot, which is a function of platelet number and fibrinogen concentration. TEG parameter Ly30 describes the stability of the blood clot in time, i.e. describes the amount of fibrinolysis.

Recorded values were exported and further analyzed in Microsoft Excel while statistical analysis was done with SPSS 17.0 (SPSS, Chicago, Illinois, USA). Statistical comparisons were done using Mann-Whitney test or $\chi^{2}$-test, as appropriate. The level of significance was set at 0.05 .

\section{RESULTS}

A total of 170 patients were included in our study. There were 100 patients operated on pump (58.8\%) and 70 patients $(41.2 \%)$ operated off-pump. Of these patients, in $20(28.6 \%)$ retransfusion of blood from cell-saver was performed. The volume of the retransfusion was approximately $480 \mathrm{~mL}$.

Thromboelastographical values are given in Table 1. It can be seen that cardiopulmonary bypass accelerates coagulation according to shortened values of reaction and clotting times ( $\mathrm{R}$ and $\mathrm{K}$ ), does not affect the clot strength and activates fibrinolysis. The fibrinolysis activation is statistically significant but may not be clinically significant because the median value of Ly30 is still in the normal range (i.e. $<7.5 \%$ ). Off-pump procedures have no substantial impact on blood coagulation assessed by thromboelastography because neither coagulation nor fibrinolytical parameters changed significantly. However, comparing values after the surgery in patient given retransfusion from cell-saver at the end of surgery with initial values in these patients $(n=20)$ the same pattern as in on-pump procedures can be seen, i.e. coagulation activation and fibrinolysis enhancement. However, the significance is again statistical but may not be clinical because the value of LY30 is still in the normal ranges. 
Table 1. Thromboelastographical values at the start of the operation (T1) and at the end (T2) in on-pump and off-pump patients with/without cell-saver.

\begin{tabular}{lcccccc}
\hline & \multicolumn{2}{c}{$\begin{array}{c}\text { On-pump } \\
\text { patients } \\
(\mathrm{n}=100)\end{array}$} & \multicolumn{2}{c}{$\begin{array}{c}\text { OPCAB patients } \\
\text { with cell-saver } \\
(\mathrm{n}=20)\end{array}$} & \multicolumn{2}{c}{$\begin{array}{c}\text { OPCAB patients } \\
\text { without cell-saver } \\
(\mathrm{n}=50)\end{array}$} \\
\hline Reaction time (s) & \multicolumn{1}{c}{$\mathrm{T} 1$} & $\mathrm{~T} 2$ & $\mathrm{~T} 1$ & $\mathrm{~T} 2$ & $\mathrm{~T} 1$ & $\mathrm{~T} 2$ \\
\hline Clot formation time (s) & $16.4 \pm 0.3$ & $22.3 \pm 0.6$ & $28.5 \pm 0.3$ & $26.3 \pm 0.5$ & $27.4 \pm 0.3$ & $27.4 \pm 0.3$ \\
Maximum amplitude (mm) & $54.4 \pm 0.3$ & $12.4 \pm 0.8$ & $16.5 \pm 0.3$ & $15.7 \pm 0.5$ & $15.5 \pm 0.1$ & $15.8 \pm 0.4$ \\
Ly30 (\%) & $2.6 \pm 0.3$ & $4.3 \pm 0.6$ & $54.5 \pm 0.3$ & $55.5 \pm 0.6$ & $54.4 \pm 0.3$ & $52.7 \pm 0.1$ \\
\hline
\end{tabular}

Abbreviations: OPCAB = Off-Pump Coronary Artery Bypass, Ly30 = clot lysis time 30 min after the maximum amplitude of the clot. Values are means \pm standard deviation.

*There is a significant difference $(P=0.01)$ between Ly30 in the group at the start of the operation (T1) and at the end of the operation (T2). †There is a significant difference $(P=0.01)$ between Ly30 in the group at the start of the operation (T1) and at the end of the operation (T2). Other comparisons within and between groups were nonsignificant.

\section{DISCUSSION}

In this prospective observational study using thromboelastography we found that both retransfusion of shed blood from cardiopulmonary bypass as well as from cellsaver in off-pump procedures activated coagulation (decreased values $R, K$, equal MA) as well as fibrinolysis (increased values Ly30). In OPCAB patients operated without cell-saver there were no differences in coagulation or fibrinolysis by TEG parameters at the start and at the end of operation.

Cardiopulmonary bypass used for heart operations in adults has an artificial surface with an area of approximately $4.2 \mathrm{~m}^{2}$ in the system and $1.6 \mathrm{~m}^{2}$ in the oxygenator. The contact of blood with this surface and with air in the standard open venous reservoir together with suctioning, ischemia and reperfusion all lead to an acute, intense, but transitory proinflammatory state. Despite anticoagulation with heparin, thrombin generation occurs and secondarily activates fibrinolysis. These disturbances - activation of both coagulation as well as fibrinolysis - can enhance blood loss after the operation and impair the function of bypasses.

In the last decade, there has been a dramatic increase in the popularity of off-pump technology in coronary artery bypass surgery. However, despite abundant evidence validating the safety and efficacy of this approach to coronary revascularization, considerable controversy persists about the long-term outcome due also to hypercoagulable state which can be induced by the operation ${ }^{3}$.

In case of surgery in which massive blood loss is expected or can be sudden, a machine for recuperation of blood - a cell-saver is advocated ${ }^{4}$. Cell-saver machines enable processing the blood removed by suction and to return it to the patient's circulation despite impact on coagulation $^{5,6}$. The filters of cell-savers can remove large particles as fat or tissue but cannot delete tissue factor and other cytokines released due to surgical trauma and affecting coagulation ${ }^{7}$. The potential advantages of perioperative blood salvage include the ready availability of large volumes of patient-compatible blood with optimal oxygen-carrying capacity free of exogenous infection ${ }^{8}$. Although the blood loss during off-pump surgery varies usually from 500 to $1800 \mathrm{~mL}$, at our institution the cellsaver is routinely used for suctioning the blood into the reservoir only. The collected blood is then processed and retransfused only when the volume is sufficiently high to warrant further costs incurred by additional setting needed for the procession. These costs are higher than the cost of one transfusion unit of foreign packed red cells.

The strength of the study is the method used to evaluate changes in coagulation and fibrinolysis - thromboelastography. This is unique because it assesses blood coagulation as a whole. It involves the effects of all factors affecting coagulation such as number and function of platelets and leucocytes, activities of coagulation and anticoagulation and fibrinolytic factors and effects of drugs, influence of temperature, acid-base balance, anaemia etc. ${ }^{9,10}$. The other major advantage of TEG is that it can show the stability of the clot in time. Thus, in contrast to conventional coagulation tests performed in plasma, TEG as a whole blood coagulation test is superior in assessing the coagulation status ${ }^{10}$.

The limitation of the study is a small number of patients in which a cell-saver was used. The decision is always based on the volume of blood loss, the hemoglobin level and circulatory status of the particular patient and with regard to economy.

\section{CONCLUSION}

In this prospective observational study it was shown that retransfusion of patient's own blood from cardiopulmonary bypass as well as from cell-saver have similar effects on coagulation. In both cases, increased fibrinolysis was discovered. On the other hand, the mutual contact of blood with endothelium of serose pericardial cavity did not significantly affect coagulation. Thus, surgery avoiding cardiopulmonary bypass and/or cell-saving techniques, i.e. 
careful physiological operating with minimal blood loss in patients with sufficiently high preoperative hemoglobin level, is the only method with no substantial impact on blood coagulation.

\section{ABBREVIATIONS}

$\mathrm{ACT}$, Activated coagulation time; $\mathrm{CABG}$, Coronary artery bypass graft; CPB, Cardiopulmonary bypass; IU, International unit; K, Clotting time; Ly30, Clot lysis time 30 min after MA was reached; MA, Maximum amplitude; OPCAB, Off-pump coronary artery bypass; R, Reaction time; TEG, Thromboelastography.

\section{ACKNOWLEDGEMENT}

Authorship contribution: TV, MH, MF literature search and manuscript writing; TV: study design; TV, MF: data collection; TV, MH, MF: data analysis and interpretation, statistical analysis; TV: final approval.

Conflict of interest statement: The authors stated that there are no conflicts of interest regarding the publication of this article.

\section{REFERENCES}

1. Bhaskar B, Dulhunty J, Mullany DV, Fraser JF. Impact of blood product transfusion on short and long-term survival after cardiac surgery: more evidence. Ann Thorac Surg 2012;94:460-7.

2. Fabre $O$, Vincentelli $A$, Corseaux D, Juthier F, Susen $S$, Bauters $A$, Van Belle E, Mouquet F, Le Tourneau T, Decoene C, Crépin F, Prat A, Jude B. Comparison of blood activation in the wound, active vent, and cardiopulmonary bypass circuit. Ann Thorac Surg 2008;86:537-41.

3. Raja SG. Hypercoagulable state after off-pump coronary artery bypass grafting: evidence, mechanism and implications. Expert Rev Cardiovasc Ther 2011;9:599-608.

4. Svenmarker $\mathrm{S}$. The inflammatory response to recycled pericardial suction blood and the influence of cell-saving. Scand Cardiovasc J 2003;37:158-64.

5. Palsgaard-Van Lue A, Strom H, Lee MH. Cellular, hemostatic and inflammatory parameter of the surgical stress response in pigs undergoing partial pericardectomy via open thoracotomy or thoracoscopy. Surg Endosc 2007;21:785-92.

6. Hunt BJ. Activation of coagulation and fibrinolysis during cardiothoracic operations. Ann Thorac Surg 1998;65:712-8.

7. Hansen E. Maschinelle Autotransfusion. Anaesthesist 2011;60:38190.

8. Ley SJ. Intraoperative and postoperative blood salvage. AACN Clin Issues 1996;7:238-48.

9. Wasowicz M, McCluskey SA, Wijeysundera DN. The incremental value of thrombolelastography for prediction of excessive blood loss after cardiac surgery: an observational study. Anesth Analg 2010;111:3318.

10. De Somer F. Understanding the delicate balance between bleeding and thrombosis: can we use it to our advantage? J Extra Corpor Technol 2012;44:P13-5. 